\title{
Le point sur les exoplanètes Une diversité inattendue
}

Anne-Marie Lagrange (anne-marie.lagrange@univ-grenoble-alpes.fr)

Institut de Planétologie et d’Astrophysique de Grenoble, Université Grenoble Alpes, BP 40700, 38058 Grenoble Cedex 9

Les exoplanètes ${ }^{(\mathrm{a})}$ sont

devenues en trente ans

un sujet majeur d'étude

en astronomie.

Les observations ont révélé une diversité importante et

inattendue dans les systèmes exoplanétaires, signe d'une grande diversité dans leurs processus de formation et d'évolution.

L'étude des exoplanètes

ne fait que commencer

et des instruments de plus

en plus spécialisés sont

en cours de réalisation

ou de conception, afin de

progresser dans ce domaine

au cours des décennies à venir.

On présente dans cet article

les principales méthodes

de détection, les résultats

majeurs obtenus,

les conséquences sur

notre compréhension

de la formation des planètes

et de leur évolution précoce, ainsi que les projets futurs

dans ce domaine.

\section{La détection des exoplanètes}

La recherche des systèmes planétaires extrasolaires n'est devenue envisageable que récemment, même si la question de l'existence d'autres mondes est très ancienne. Le désir d'exploration et l'imagination étaient bien présents, mais les moyens techniques n'étaient pas suffisants pour permettre de découvrir des planètes extrasolaires. L'observation directe, moyen le plus naturel qui soit, est restée longtemps impossible à cause du fort contraste de luminosité et de la très faible séparation angulaire entre l'étoile et la planète. En effet, ce contraste, dans le domaine optique, est, par exemple, de plusieurs milliards entre le Soleil et la Terre, et de plusieurs dizaines de millions entre le Soleil et Jupiter. Par ailleurs, vue d'une étoile du voisinage solaire située à 10 parsecs $^{(b)}$, la Terre ne serait séparée que de 0,1 seconde d'angle du Soleil, et Jupiter que de 0,5 seconde. C'est pourquoi les astronomes se sont tournés dès les années 1980 vers des techniques indirectes, visant à détecter et caractériser des variations de certains signaux des étoiles entourées de planètes.

Les variations peuvent concerner :

- la position des étoiles (mesure du mouvement d'oscillation dans le plan du ciel du photo-centre de l'étoile par rapport au centre de gravité étoile-planète, méthode astrométrique) ;

- leur vitesse radiale (mesure des variations de vitesse projetée de l'étoile sur la ligne de visée entre l'observateur et l'étoile, qu'on détermine par effet Doppler sur certaines raies du spectre de l'étoile);

- leur luminosité.

Les deux premières méthodes reposent sur l'application des lois de Kepler ${ }^{(c)}$. Si aucune exoplanète n'a encore été découverte en astrométrie, le satellite GAIA de
l'Agence Spatiale Européenne devrait permettre dans les années à venir de détecter plusieurs milliers d'exoplanètes géantes par cette méthode. La méthode des vitesses radiales (fig. 1) a permis les premières détections dans les années 1990 [1] ; à ce jour, on compte plusieurs centaines de planètes détectées et caractérisées par cette méthode. La troisième méthode vise à mesurer des baisses régulières de flux stellaire dues au passage d'une planète sur la ligne de visée. Cette "méthode de transit " a connu son apogée récemment, grâce aux observations depuis l'espace, avec notamment le satellite Corot du CNES, lancé en 2006, et le satellite Kepler de la NASA, lancé en 2009. Ce dernier a révélé plus de 2000 exoplanètes.

Les méthodes des vitesses radiales et des transits ont permis de trouver des planètes d'abord géantes, puis au fur et à mesure des progrès techniques ou dans le traitement des données, des planètes plus petites, jusqu'à atteindre des planètes de masses ou de rayons à peine plus grands que celle/ celui de la Terre. Ces méthodes permettent de détecter des exoplanètes plutôt proches de leur étoile. En effet, les détections et caractérisations nécessitent de suivre les variations de vitesses radiales ou photométriques sur des périodes de temps au moins égales à la période orbitale de la planète (un an pour la Terre autour du Soleil et douze ans pour Jupiter). Plus spécifiquement, la méthode des vitesses radiales permet de mesurer la quantité M.sin(i) où i représente l'inclinaison du plan orbital de la planète par rapport à la ligne de visée, et d'en déduire une limite inférieure de sa masse $\mathrm{M}$, alors que la méthode des transits permet de mesurer le rayon des planètes (déduit de la surface de l'étoile occultée au cours du transit), mais pas sa masse. 


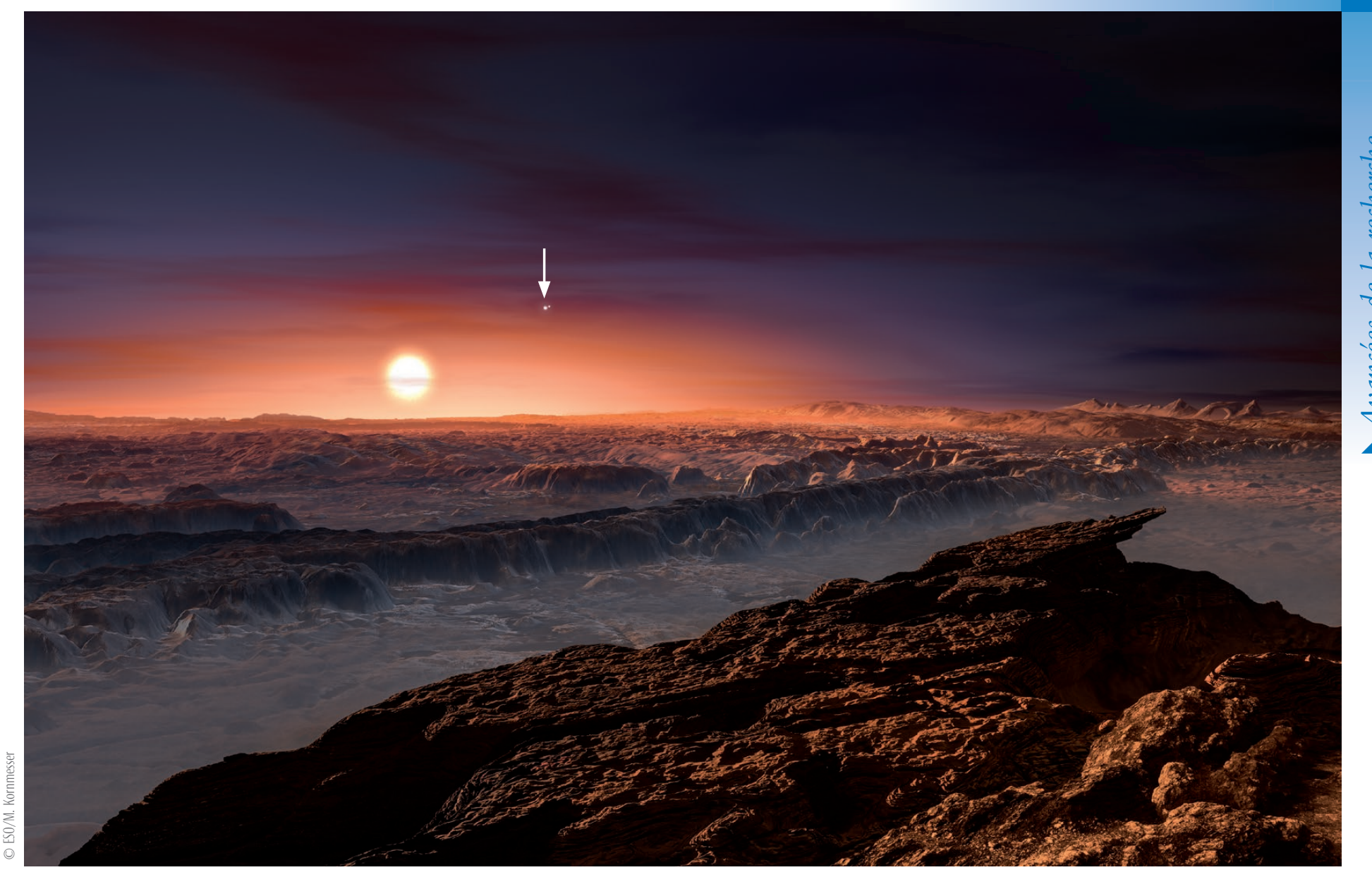

Impression d'artiste de la surface de la planète Proxima Centauri b, en orbite autour de la naine rouge Proxima Centauri, l'étoile la plus proche du système solaire. L'étoile double Alpha Centauri AB (indiquée par une flèche) apparait aussi sur l'image. Proxima b est un peu plus massive que la Terre et se situe dans la zone « habitable " autour de Proxima Centauri, où la température est compatible avec l'existence d'eau liquide à la surface.

On notera enfin l'existence d'autres méthodes de détection indirecte, comme celle des lentilles gravitationnelles [3] qui permet de détecter et caractériser les exoplanètes une fois, mais ne donne pas la possibilité de les réobserver, et la méthode, très spécifique, dite de chronométrage des pulsars.

Le lecteur intéressé par les techniques de détection peut se reporter aux références données page 24 .

L'imagerie directe est, a priori, bien plus adaptée que les méthodes indirectes pour détecter des planètes plus éloignées de leur étoile. Elle nécessite l'utilisation (i) de télescopes de grand diamètre (un miroir primaire de diamètre $\mathrm{D}$ donnant un pouvoir de résolution égal à $1,22 \lambda / \mathrm{D}$ à la longueur d'onde $\lambda$ ) ; (ii) depuis le sol, des dispositifs d'optique adaptative permettant de corriger en temps réel des effets de la turbulence atmosphérique ${ }^{(\mathrm{d})}$; et (iii) de coronographes, dispositifs optiques permettant d'occulter la plus grande partie de la lumière de l'étoile afin de détecter le
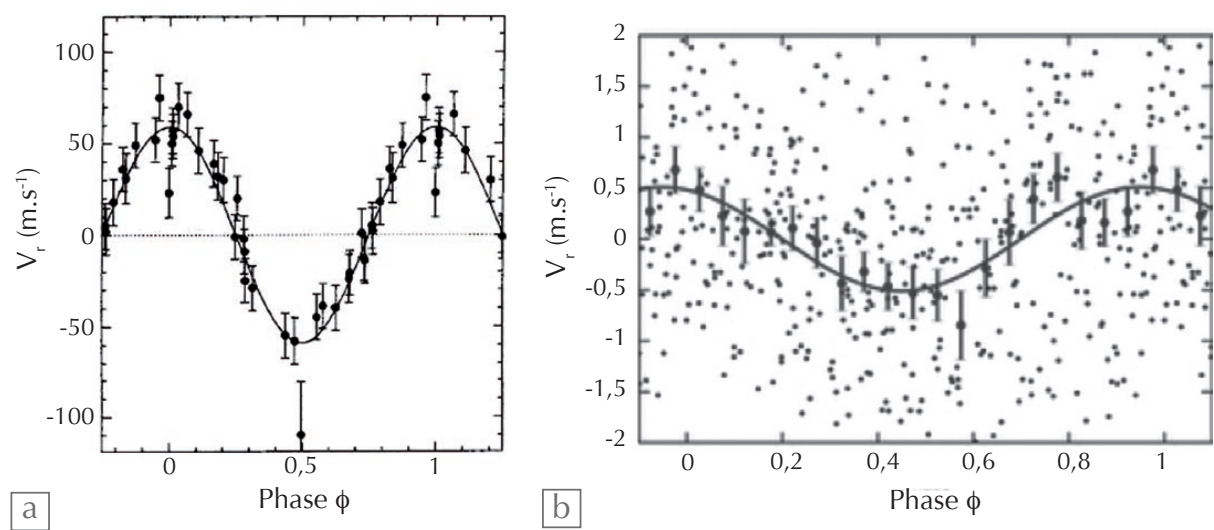

1. Deux détections d'exoplanètes, séparées de 20 ans, obtenues par la méthode des vitesses radiales. (a) 51 Peg b, de 0,47 fois la masse de Jupiter et sur une orbite circulaire de rayon 0,052 unités astronomiques (ua) ${ }^{(b)}$, détectée en 1995 dans la constellation de Pégase (Mayor et Queloz [1]).

(b) Proxima Cen b, de 1,3 fois la masse de la Terre et de demi grand-axe orbital 0,05 ua, détectée en 2016 (Anglada-Escude et al., [2]).

Les vitesses radiales $V_{r}$ sont données ici en fonction de la phase $\phi$ de la révolution de la planète, chaque point représentant une mesure. Les ajustements par des orbitales kepleriennes sont indiqués en traits pleins. On notera les différences d'amplitude des ajustements orbitaux : de l'ordre de $50 \mathrm{~m} / \mathrm{s}$ pour la planète géante 51 Peg b, et de l'ordre de $10 \mathrm{~cm} / \mathrm{s}$ pour la planète de petite masse Proxima Cen b. On notera également la dispersion des points de mesure dans le cas de Proxima Cen b. 51 Peg b tourne autour d'une étoile semblable au Soleil, tandis que Proxima Cen b tourne autour d'une étoile plus froide (naine rouge), Proxima Centauri, qui est l'étoile la plus proche $(1,3$ pc) du système solaire. 

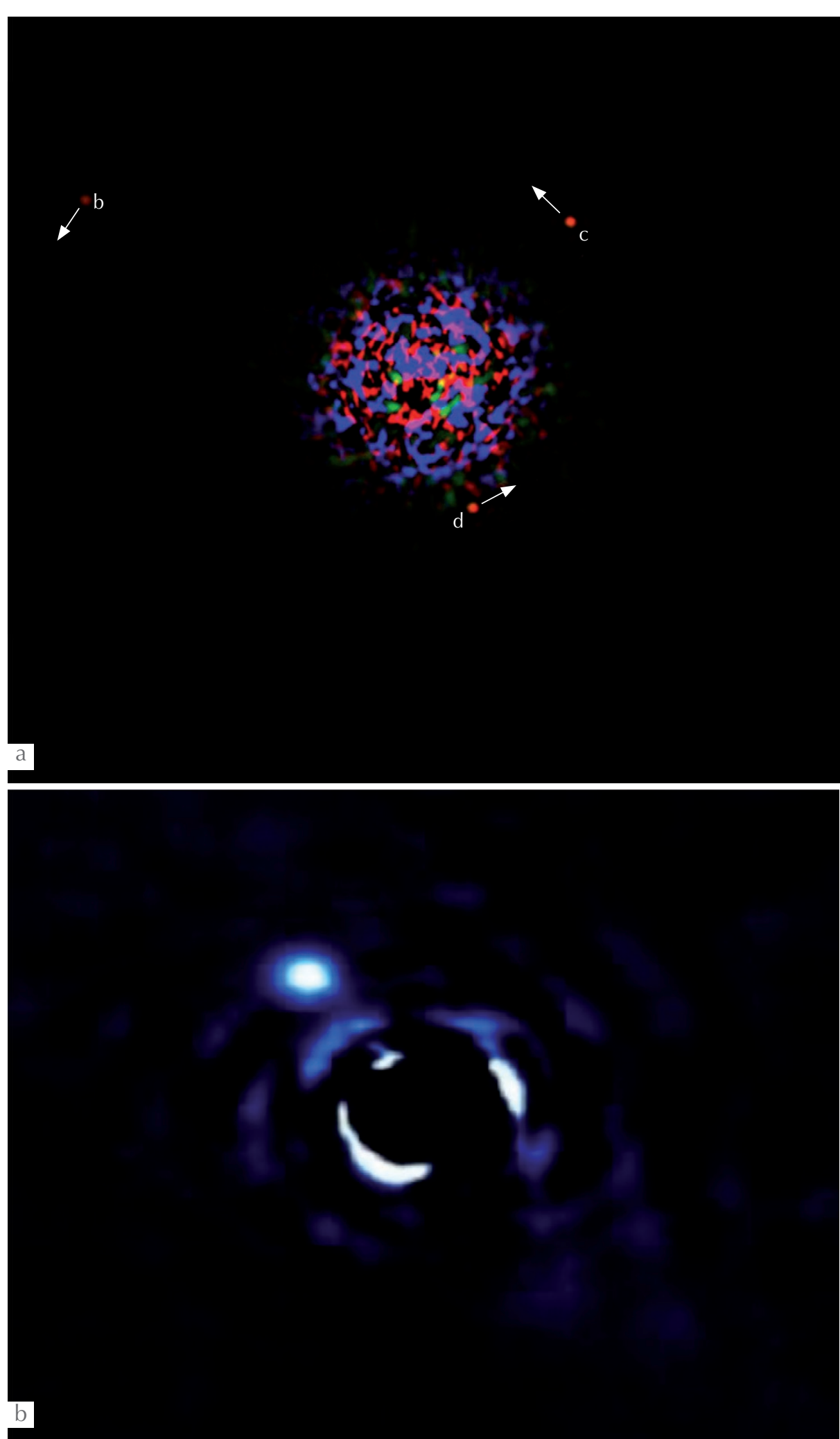

2. Exemples d'images directes d'exoplanètes.

(a) Image de la découverte du système planétaire autour de l'étoile HR8799, située à 39 pc du Soleil (Marois et al. [4]). (b) Image de la planète Beta Pictoris b, dont l'étoile est située à 19 pc du Soleil (Lagrange et al., [5]).

Dans ces images, le Nord est vers le haut et l'Est vers la gauche. Les séparations des planètes b, c et $d$ autour de HR8799 sont d'environ 68, 42 et 27 ua ; la distance de Beta Pictoris b de son étoile est de 9 ua. Noter que les étoiles elles-mêmes ne sont pas visibles, car elles sont soit occultées par un masque coronographique, soit éliminées au cours du traitement d'image effectué pour faire « ressortir » les exoplanètes. Seuls des résidus de réduction sont visibles au centre des images.

\section{$>>$}

faible signal de la planète. Avec les systèmes d'optique adaptative développés depuis les années 2000 et installés sur des télescopes au sol de la classe des dix mètres de diamètre, les premières images d'exoplanètes ont été obtenues (fig. 2).

De nouveaux instruments dédiés à la détection des exoplanètes, plus sophistiqués, comprenant des systèmes d'optique adaptative extrême, couplés à de meilleurs coronographes, ont été mis en service très récemment au Chili, sur les télescopes de huit mètres de diamètre du Very Large Telescope de l'Observatoire Européen Austral (ESO), d'une part, et de l'observatoire GEMINI Sud de l'AURA (Association of Universities for Research in Astronomy), d'autre part. Offrant des contrastes d'environ $10^{-6}$ à 0,3 seconde d'angle, ils devraient permettre de faire l'image de quelques dizaines de planètes jeunes en orbite à quelques unités astronomiques d'étoiles du voisinage solaire.

Deux observations, séparées de quelques mois seulement, sont suffisantes pour identifier une planète. (Caractériser complètement ses propriétés orbitales nécessite bien sûr un suivi beaucoup plus long, de l'ordre de la période orbitale de la planète.) Il convient enfin de noter que la masse et la température effective de la planète ne sont pas directement mesurées ; elles sont estimées en utilisant des relations massebrillance, ou par comparaison entre des spectres observés et des spectres synthétiques dérivés de modèles atmosphériques qui, jusqu'à présent, manquent de calibrateurs. Pour les planètes jeunes (âgées de quelques millions ou de quelques dizaines de millions d'années) ${ }^{(e)}$ actuellement accessibles à l'imagerie, température et masses dépendent de manière cruciale de leur âge, un paramètre particulièrement difficile à estimer de manière précise.

\section{Le bestiaire des exoplanètes}

Les astronomes ont coutume de représenter les détections des exoplanètes dans un diagramme représentant leur masse en fonction du demi grand-axe de leur orbite elliptique (fig. 3). Cette représentation permet d'identifier différentes familles d'exoplanètes, et en particulier :

- Les "Jupiters chauds ", qui sont des planètes de masse ou de rayon proches de ceux de Jupiter, de période orbitale de 1 à 100 jours. Ces planètes ont été les premières découvertes, car elles sont 
- Transits

- Vitesses radiales

- Microlentilles

- Imagerie directe

- Système solaire

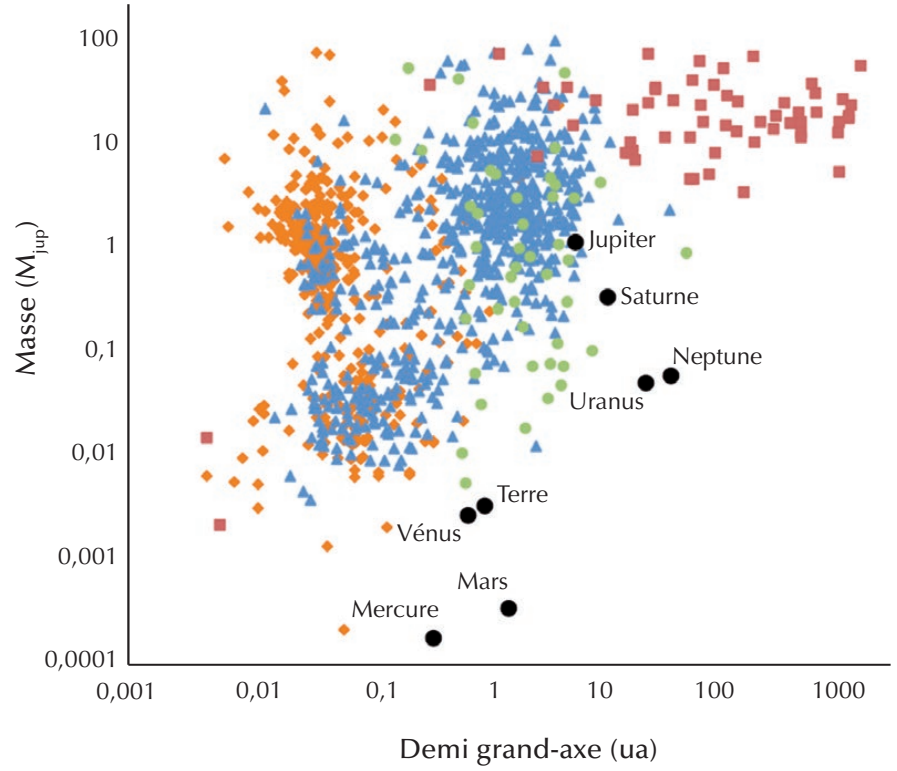

Demi grand-axe (ua)

3. Graphe représentant les masses et demi grand-axes des exoplanètes détectées par diverses méthodes : vitesses radiales, transits, microlentilles et imagerie. Pour comparaison, les planètes du système solaire sont indiquées par des cercles noirs.

bien plus faciles à détecter que des planètes de période plusieurs milliers de jours. Ces courtes périodes indiquent (lois de Kepler) que les planètes orbitent à quelques rayons stellaires seulement de leur étoile. Leur atmosphère est donc très chaude (plus de 1000 degrés), ce qui a donné leur nom de "Jupiters chauds ». La présence de planètes géantes aussi proches de leur étoile n'était pas prévue par le scénario de formation des planètes géantes du système solaire. On admet aujourd'hui que ces planètes se sont formées plus loin de leur étoile, comme les planètes géantes du système solaire, par accrétion de gaz sur un noyau rocheux, au-delà de la ligne de glace ${ }^{(f)}$, et qu'elles ont ensuite migré vers leur étoile suite à des interactions avec le disque protoplanétaire dans lequel elles se sont formées, ou bien encore suite à des interactions gravitationnelles entre plusieurs planètes au sein de systèmes multiples. Si les Jupiters chauds sont nombreux dans le diagramme (masse, demi grand-axe) par rapport, par exemple, aux Jupiters situés à une unité astronomique ou plus, ils ne sont pas forcément très fréquents. Leur grand nombre dans ce schéma est lié au fait qu'ils sont plus faciles à détecter que leurs homologues plus lointains.

- Des « super-Terres »: cette classe de planètes, de plusieurs masses terrestres, était inattendue car elle n'est pas observée dans le système solaire. Certaines de ces planètes pourraient être entourées d'eau liquide (" planètes océans ").

- Des planètes géantes massives, orbitant à plusieurs dizaines, voire plusieurs centaines d'unités astronomiques de leur étoile. Ces planètes lointaines représentent un défi pour le scénario traditionnellement admis de formation des planètes géantes du système solaire, dans lequel celles-ci se forment au sein d'un disque protoplanétaire dense, par accrétion de gaz sur un noyau rocheux, à quelques unités astronomiques seulement de leur étoile. Si certaines de ces planètes lointaines pourraient avoir été formées près de leur étoile et avoir ensuite été éjectées par interaction gravitationnelle avec d'autres corps présents autour de l'étoile (planètes, naines brunes, étoiles), certaines ont du mal à être expliquées par un tel scénario. Des mécanismes alternatifs sont donc envisagés : la formation par effondrement gravitationnel du disque protoplanétaire, ou bien la formation par effondrement de condensations à l'intérieur de nuages protostellaires. Ce dernier scénario est celui couramment admis pour expliquer la formation des étoiles dans des systèmes doubles ou multiples. Très récemment, un système d'étoiles multiples vraisemblablement formé par fragmentation d'un disque gravitationnellement instable a été découvert. Ceci indique peut-être une sorte de continuité entre les formations planétaire et stellaire.

La diversité en demi grand-axes des orbites des planètes s'accompagne d'une diversité en termes d'excentricités, mais également en termes d'inclinaisons par rapport au plan de l'écliptique, et de direction de rotation par rapport à la rotation de l'étoile (orbites rétrogrades). Ceci constitue une nouvelle différence par rapport aux huit planètes bien connues du système solaire, qui ont toutes des orbites circulaires ou très peu excentriques, tournant dans la même direction et très peu inclinées $^{(\mathrm{g})}$. De nouveau, des perturbations gravitationnelles avec d'autres corps présents autour des étoiles, conduisant à l'éjection de ces planètes de leur orbite initiale, pourraient expliquer ces particularités. Enfin, on notera aussi une très grande diversité dans les architectures des quelques 500 systèmes multiplanétaires découverts à ce jour.

La caractérisation des planètes va au-delà de la détermination de leur masse et de leurs propriétés orbitales. Les propriétés atmosphériques (composition, structure, température), de même que la structure interne des planètes font aussi l'objet de recherches intensives. Lorsque la masse et le rayon, donc la densité moyenne des 
planètes sont connus, il est possible en effet de contraindre un peu la nature (gazeuse, rocheuse) de ces dernières et, dans une certaine mesure, leur structure interne (présence ou absence d'un noyau dense, présence d'eau, etc.).

Des informations sur la composition et la température des atmosphères peuvent être obtenues grâce à des études photométriques ou, mieux, à des études spectroscopiques, pour les planètes en transit (donc proches de leur étoile) ou bien, au contraire, pour les planètes lointaines observées en imagerie. Dans les deux cas, il s'agit pour le moment d'atmosphères chaudes (typiquement plus de 1000 kelvins). Les atmosphères des quelques exoplanètes étudiées se révèlent variées et complexes. Selon les conditions physiques, des brumes ou des nuages de poussières peuvent être présents. Des phénomènes de variabilité temporelle (on parle déjà de "météo " des exoplanètes) sont envisagés.

\section{Conclusion et perspectives}

Au début de 2017, on recensait plus de 3000 exoplanètes confirmées. Mais, malgré les avancées impressionnantes des trois dernières décennies, nos connaissances restent bien limitées. Nous ne sommes pas encore en mesure, par exemple, de détecter, s'ils existent, des systèmes exoplanétaires analogues au système solaire. Même si nous savons qu'au moins 5\% des étoiles de type solaire abritent une planète géante, et beaucoup plus abriteraient des planètes telluriques, nous ne connaissons pas encore le véritable taux d'occurrence des planètes ; nous ne savons pas à quel point ce taux et les propriétés des planètes dépendent des propriétés des étoiles mères. La caractérisation des planètes, en particulier l'étude de leur atmosphère et de leur intérieur, est encore restreinte à un très petit nombre d'objets, et les connaissances sont particulièrement limitées.
Un des objectifs des astronomes et des astrobiologistes est de trouver, un jour, des signatures de vie sur des planètes telluriques évoluant dans la zone "habitable " des étoiles, cette zone dans laquelle l'eau à la surface de la planète serait sous forme liquide $^{(\mathrm{h})}$. Quelques planètes de type tellurique ont été trouvées dans la zone habitable d'étoiles froides (naines $M$, comme par exemple Proxima du Centaure), mais pas encore autour d'étoiles semblables au Soleil (de telles planètes étant plus difficiles à détecter). Il est aujourd'hui impossible de donner une liste des conditions nécessaires et suffisantes à l'apparition et à la durabilité de la vie sur ces planètes, ce qui rend l'objectif de recherche de signature de vie encore assez flou.
Les télescopes du futur, en particulier le télescope spatial James Webb, successeur du télescope Hubble, et dont le lancement est prévu en 2018, devraient permettre de progresser de manière significative dans la caractérisation des atmosphères des exoplanètes observées par la méthode de transit, en commençant par les géantes et en continuant par des planètes de type Neptune ou super-Terre. À partir de 2025, des télescopes géants au sol, de 30 à $40 \mathrm{~m}$ de diamètre, devraient, eux, permettre de réaliser des images de super-Terres dans la zone " habitable » de certaines étoiles et de caractériser, au moins de manière sommaire, leur atmosphère. Nul doute que les prochaines décennies seront riches de nouvelles découvertes dans le domaine des exoplanètes.

(a) Une exoplanète (ou planète extrasolaire) est définie par l’Union Astronomique Internationale comme un astre de masse inférieure à la masse limite permettant la fusion du deutérium (soit environ treize fois la masse de Jupiter dans le cas de corps dont la fraction en éléments hors hydrogène et hélium est identique à celle du Soleil), en orbite autour d'étoiles ou de restes d'étoiles. On notera que les corps de masse inférieure à cette limite non liés à des étoiles ("free floating planets") ne sont pas des exoplanètes.

(b) 1 parsec (pc) correspond à la distance à laquelle une unité astronomique (ua) est vue sous un angle d'une seconde d'arc. $1 \mathrm{pc} \sim 3,086 \times 10^{16} \mathrm{~m} \sim 3,26$ années-lumière.

Une unité astronomique (ua) est égale à la distance Terre-Soleil, soit $149597870700 \mathrm{~m}$ (près de 150 millions de km).

(c) Les lois de Kepler caractérisent le mouvement orbital d'une planète autour de son étoile, selon une ellipse dont l'étoile occupe l'un des foyers. De manière symétrique, la planète induit un mouvement de l'étoile, de période égale à la période de révolution de la planète, et d'amplitude bien plus petite que celle de la planète.

(d) Les turbulences atmosphériques déforment le front d'onde plan incident provenant d'un astre lointain et vu comme un objet ponctuel, provoquant ainsi un étalement du signal au foyer de l'instrument selon une tache appelée tache de "seeing", dont la taille est caractéristique de l'état de l'atmosphère et pas de la taille du télescope, comme ce serait le cas pour un télescope spatial. Dans des sites de qualité moyenne, le diamètre de la tache de "seeing" peut être de plusieurs secondes d'angle, tandis que dans les meilleurs sites connus actuellement, il peut être aussi petit que 0,3 secondes. Hors atmosphère, la taille de l'image est inversement proportionnelle au diamètre du télescope. Spatialiser de très grands télescopes est toutefois onéreux et compliqué.

(e) Les exoplanètes peuvent être "vues » a priori soit grâce à leur émission thermique, soit grâce à la lumière qu'elles réfléchissent de leur étoile. Les instruments actuels ne permettent que d'imager celles qui sont suffisamment chaudes pour être détectées grâce à leur émission thermique. Ce sont donc des planètes géantes jeunes, pas totalement refroidies depuis le moment de leur formation.

(f) La ligne de glace de l'eau est la distance à l'étoile au-delà de laquelle l'eau est présente sous forme condensée. La même notion peut s'appliquer à d'autres molécules volatiles (par exemple le dioxyde de carbone, le monoxyde de carbone, le méthane, etc.).

(g) Récemment, l'existence d'une neuvième planète dans le système solaire a été inférée à partir d'études dynamiques; elle n'a toutefois pas encore été observée ni caractérisée.

(h) Attention, la présence d'eau n'étant certainement pas une condition suffisante à la présence de vie, le terme " habitable " peut prêter à confusion.

\section{Références}

1• M. Mayor et D. Queloz, "A Jupiter-mass companion to a solar mass star", Nature 378 (1995) 355-359.

2 $\bullet$ G. Anglada-Escude et al., "A terrestrial planet candidate in a temperate orbit around Proxima Centauri”, Nature 536 (2016) 437.

3• J.P. Beaulieu et A. Cassan, Reflets de la physique 9 (2008) 7-11.

4 C. Marois et al., "Direct Imaging of Multiple Planets Orbiting the Star HR 8799", Science 322 (2008) 1348-1352.

5• A.M. Lagrange et al., "A Giant Planet Imaged in the Disk of the Young Star $\beta$ Pictoris”, Science 329 (2010) L5.

\section{Pour en savoir plus}

- A.-M. Lagrange et P. Léna, «Exoplanètes ou planètes extrasolaires », Encyclopédie Universalis (2016).

- «L’Encyclopédie des planètes extrasolaires », Observatoire de Paris (multilingue), http://exoplanet.eu/

- NASA Exoplanets Archives, Californian Institute of Technology, http://exoplanetarchive.ipac.caltech.edu/ 